\title{
Design of Intelligent Rehabilitation Service System Based on “Internet Plus”
}

\author{
Ao Zhang, Shuo Wang ${ }^{*}$, Yunjia Zhang \\ Qingdao University of Technology, Qingdao, Shandong Province, China \\ *Corresponding author: Shuo Wang
}

Keywords: Combination of medical and nursing, Intelligent health, Internet of things, Blockchain, Cloud computing

\begin{abstract}
The health care service system uses modern technology such as computer network technology and the Internet of Things, through various sensors, full monitoring, and real-time positioning functions to monitor all kinds of information of the elderly at anytime, anywhere, and comprehensively. Under the linkage, medical and nursing integration, wisdom and well-being, to provide timely, convenient and accurate medical services for the elderly, and ultimately provide integrated medical and nursing services by integrating medical services, life care services, health rehabilitation and hospice care So as to meet the overall needs of the elderly for the elderly.
\end{abstract}

\section{Introduction}

Nowadays, population aging is a worldwide problem. my country is one of the countries with a relatively high population aging in the world. It has the largest number of elderly people, the fastest aging rate, and the inversion of urban and rural aging. The task of coping with population aging is the heaviest.[1] By the end of 2019, the national population of people over 60 years of age will increase to about 255 million people, accounting for $18.1 \%$ of the total population. In order to solve this world problem, according to the traditional virtues of China, more elderly people can "have a place to live, and have a care for the old”. In response to national policies, the model of combining medical care and convalescence is promoted. Maintenance service system.

\section{Design of Smart Health Service System}

\subsection{Functional Design of Health Care System}

According to the overall architecture design, the community smart pension platform can be divided into smart home systems, remote care systems, emergency help systems, and medical health systems according to their functions. These functions are based on cloud computing platform data and provide personalized services for the elderly to achieve the purpose of smart pension. [2]

\subsubsection{Smart Home System Functions}

Smart home provides a safe, reliable and comfortable living environment for the elderly. According to the overall frame structure, smart home functions include intelligent control of home appliances, automatic monitoring and alarming, smart security, remote video communication, and air quality control, as shown in Figure 1.

\section{Smart Home System}

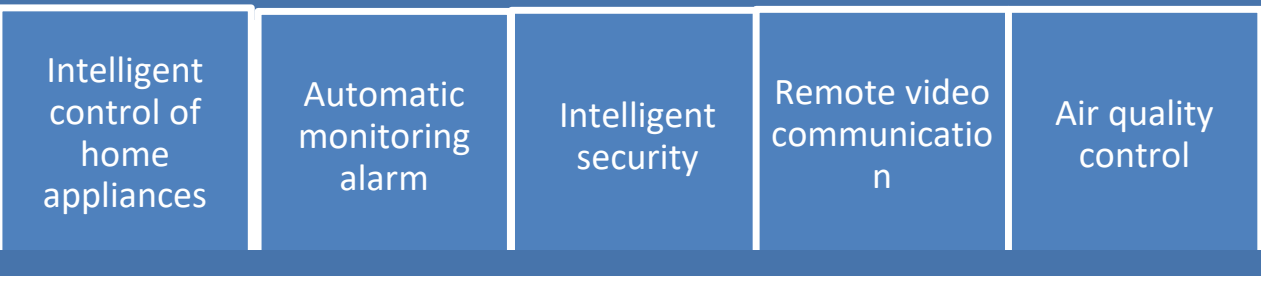

Fig.1 Functional Diagram of the Smart Home System 
(1)Intelligent control appliances

Such as intelligent control of lights, intelligent control of air conditioners, intelligent control of humidifiers and purifiers. When the indoor lighting, temperature, and humidity do not reach a certain amount of standards, the smart home system can automatically control the lighting, air conditioning, humidifier, etc. to adjust the indoor temperature, humidity, lighting, etc., to provide a comfortable indoor environment for the elderly.

(2)Automatic monitoring alarm

When the temperature and smoke in the room reach a certain value, the service terminal will automatically network alarm and notify the community pension service center and elderly children. [3] There are emergency help buttons in the bedside, bathroom and living room. The elderly can call for help when they are in danger. The system will immediately call the police to remind their children, community pension service center and property security.

(3)Intelligent security

There are anti-theft alarms, door and window monitoring and intrusion alarms. When a security incident occurs, you can promptly notify your children, community and property security. In addition, children can open and close the door remotely to control the doors and windows to prevent the elderly from forgetting to close the door.

(4)Remote video communication

Remote video communication mainly means that children can monitor the implementation of the elderly at home anytime and anywhere through their mobile phones to ensure the safety of the elderly and prevent accidents. Children and old people can also interact with their children through videos, which is very convenient.

(5)Air quality control

Indoor air quality is very important to the health of the elderly. Children can check the formaldehyde, dust, PM2.5 and other conditions in their homes in real time through the indoor air quality monitoring system. When it is found that the harmful gas exceeds the standard, the children can be reminded in time, and the ventilating fan, exhaust fan, air purifier, etc. can be automatically adjusted to improve the indoor air quality and provide a healthy indoor environment for the elderly.[4]

\subsubsection{Remote Care System Functions}

The services of remote care include remote detection, emergency call, health consultation, and rehabilitation training functions. Remote care enables the elderly to get timely care and health guidance, saves medical and health resources, provides long-term monitoring for the elderly, and improves the quality of life of the elderly . The specific functions of remote care are shown in Figure 2.

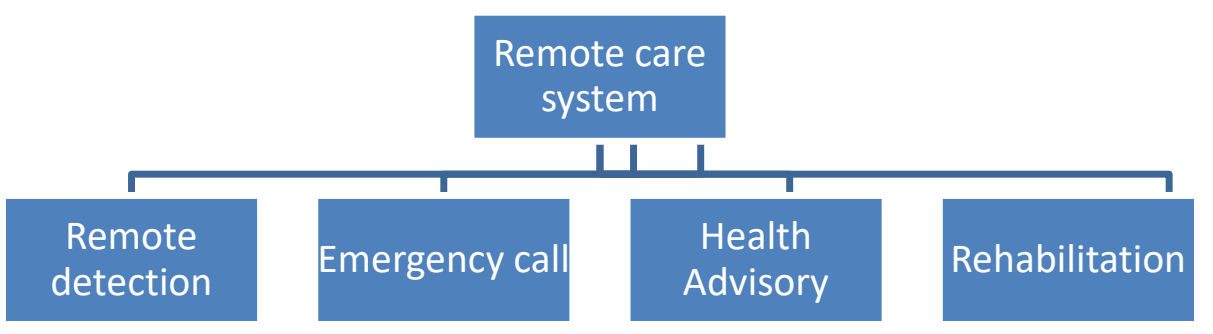

Fig.2 Functional Diagram of the Remote Care System

(1) Remote detection

The wearable device can detect the heartbeat, heart rate, breathing and other conditions of the elderly and send them to the data center of the platform. When the elderly's heartbeat, heart rate, breathing and other vital signs are abnormal, the data center will report to the community pension service center. Send an alarm signal to the elderly and children, and send the alarm information to them.

(2) Emergency call

Set up the monitoring of accidents, falls, etc., the elderly can call emergency events. If an 
elderly person encounters an emergency, he can call the help center through the one-button help function on the mobile phone or smartphone ring. After receiving the distress signal, the data center will promptly send the alarm information to the community pension service center and the children of the elderly.

(3) Health consultation

Medical staff can provide the elderly with knowledge and psychological guidance on disease health, including consultation on disease risk factors and prevention, and timely answer the disease and health questions raised by the elderly. Service personnel such as the community can provide the elderly with anti-fraud and other safety prevention education knowledge to improve the elderly's safety prevention awareness.[5]

(4) Rehabilitation training

Platform helps the elderly recover from illness through remote guidance. The elderly can watch and participate in rehabilitation through customized video software. Through the computer-based online rehabilitation model of medical staff and the elderly, the rehabilitation compliance of the elderly is improved.

\subsubsection{Emergency Help System Function}

The elderly, especially those living alone, sometimes encounter some very dangerous things, such as forgetting to turn off the gas, bumping up and down the stairs, etc. The emergency help function of this platform can use emergency call extensions and smart wearable positioning devices, Passive infrared detectors and other instruments to ensure that the elderly can be rescued as soon as they encounter danger. The specific functions are shown in Figure 3.

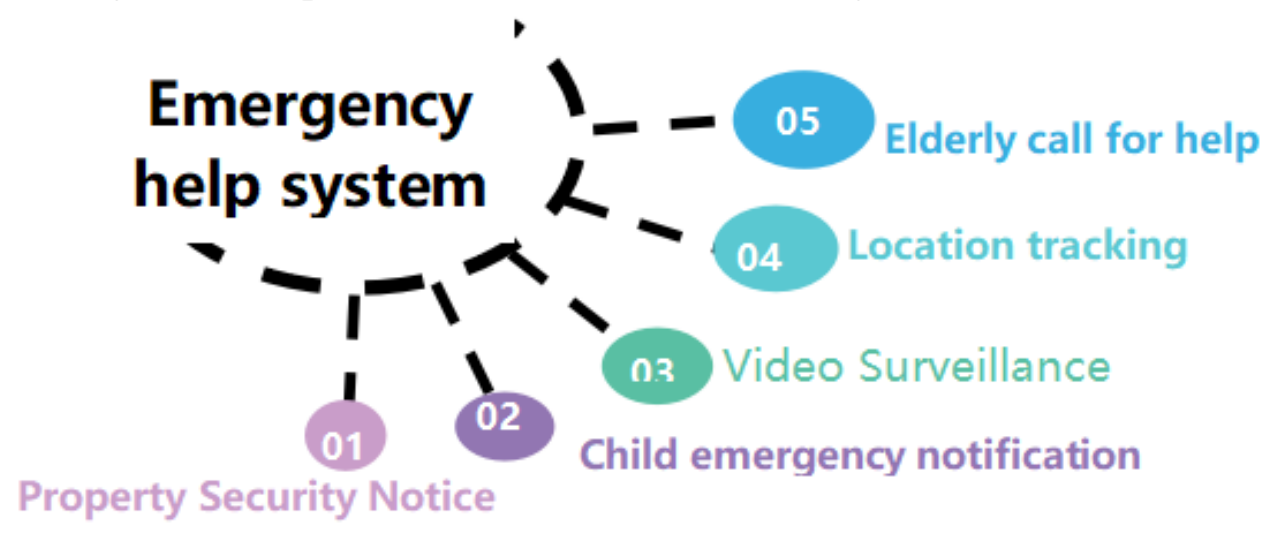

Fig.3 Emergency Help System Function Diagram

The elderly can call for help by binding a mobile phone or a phone. When the platform receives a call for help, they can contact the family of the elderly as soon as possible and send community personnel to help the elderly. Actual situation, determine whether to dial 110 or 120 .

The smart wearable device can feedback the position information of the elderly to the platform in time through GPS positioning technology. When the elderly encounter a danger, they can immediately determine the location of the elderly and rescue them in time.[6]

The video monitoring function feeds back the environmental data of the elderly to the platform through the mobile communication network transmission, and the cloud computing platform can intelligently start emergency resources.

\subsubsection{Medical and Health System Functions}

Medical health is the most important function of the smart pension platform. According to the overall framework of the system, the specific functions are shown in Figure 4, including regular physical examination, appointment registration, online consultation, health information feedback and other functions. 


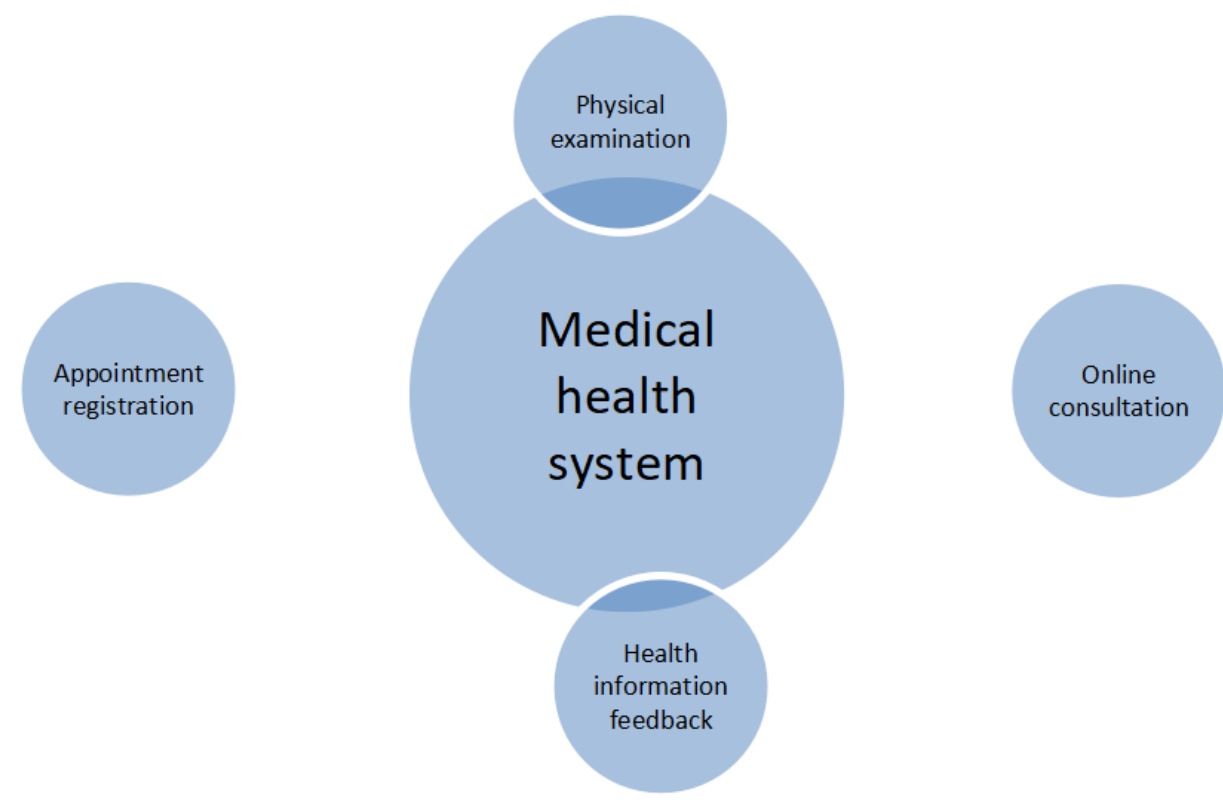

Fig.4 Medical Health System Function Diagram

The community service center regularly arranges for the elderly in the community to perform physical examinations in batches and store the examination results in the elderly's health files.

When there is a disease pre-examination in remote monitoring, or the elderly feel that they are suffering from illness, they can make an appointment registration through the smart gateway, and they can get the diagnosis results at home after the diagnosis results are out, or they can make an online consultation through an appointment network doctor, You can get the diagnosis without leaving home. The diagnosis will also be stored in the elderly's health file.

The community pension service center will often upload various types of health information to the smart pension platform, including videos and articles on dietary recommendations for the elderly, exercise methods, disease control, etc., and delineate various recommended tags for these videos and articles. The elderly can pass The TV version of the home care software of the cloud computing platform can view various types of care videos and articles by itself. The smart care platform will also recommend and screen related information for the elderly based on the fusion results of the elderly data.

\subsection{Construction of Health Care System}

According to the smart pension service system, based on the Internet of Things, cloud computing, mobile Internet, big data and other technologies, the smart pension service system architecture is proposed. The platform is composed of six parts: the Internet of Things layer, equipment layer, platform layer, system layer, users and technical support.

The IoT layer is mainly composed of a variety of smart home devices, video devices, and wearable devices based on sensor technology. Among them, the technologies used to ensure the safety of the elderly at home are divided into five categories: general adaptive technology, passive environmental sensing technology, Life assistance technology, mobile management technology, and home equipment technology must be reliable, low-invasive, and cost-effective. Existing studies have analyzed the cost-effectiveness of implementing IoT technology in elderly care. This study quantitatively evaluates the use of smart home technology and video access technology in home care, demonstrating the cost-effectiveness of smart home technology. The cost of IoT equipment continues to decrease with the advancement of technology, and its application in smart elderly care has good prospects.

The cloud computing platform layer provides unified management of data, including cloud storage and cloud computing. Store and backup data in the cloud, provide a unified interface for data upload and sharing; perform intelligent calculations on the data, establish models for long-term evolution analysis, generate automatic health status analysis reports, intelligent response strategies 
for environmental changes, intelligent start-up emergency resources, and automatic matching Life service needs.

The system layer provides multiple service systems for each of the elderly care services based on the data and intelligent computing results provided by the cloud computing platform layer, including smart home systems, remote care systems, user feedback systems used by elderly users and relatives, and community service agencies. Property management system, interactive communication system, emergency rescue system, medical health system used by medical institutions, customized service system used by third-party pension institutions, and pension security system used by the government. The application layer program adopts B / S architecture. Users can use popular smartphones as terminals to reduce implementation costs. At the same time, the application layer makes special designs for the unique visualization needs of the elderly group.

The system is built on the basis of SOA framework technology, using Web semantic technology to seamlessly connect various service systems, and provide corresponding functional modules for users through identity authentication technology; the platform also uses technologies such as mobile Internet, smart sensing, and GPS positioning.

\section{Conclusion}

Severe aging trends have brought tremendous old-age pressure to every family. Today, information technology continues to bring profound changes to all areas of society. Using information technology to optimize the allocation of old-age resources and innovative old-age service models are the only way to deal with the old-age dilemma. The transition from traditional old-age care to smart old-age care includes both the intelligent transformation of a single old-age care method and the process in which the main bodies of old-age care services collaborate with each other and exert their respective advantages to meet multi-level needs. Based on the existing research and practice results, the concept of smart old-age care is reinterpreted, and a fully integrated smart old-age care service system is proposed. Based on this, a smart old-age care service technology platform is constructed to provide guidance for the development of the smart old-age service industry in China. The system uses modern technology such as computer network technology and the Internet of Things, through various sensors, full monitoring, and real-time positioning functions, combined with traditional family, community, institutional old-age care and other traditional old-age care methods to provide a close combination to meet the material Spiritual needs, to provide more quality and effective services for the elderly.

\section{References}

[1] Song, X. The old-age Wisdom Becomes the New Model of the Old-Age Pension. Jushe, no. 08, pp. 16-17, 2020.

[2] Zhang, Z.L. Research on the Model of Smart, Healthy Old-Age Care under the Background of Big Data. Smart City, vol. 6, no. 04, pp. 19-20, 2020.

[3] Chen, H.C., Qian, D.D., Gu, Z.Y., et.al. Innovation and Development of Student Health File Management Based on Blockchain Technology. China Collective Economy, no. 35, pp.147-150, 2019.

[4] Qiu, Y., Wang, C., Qi, K.Y., et.al. A Review of Intelligent Health Research: Systems from the Cloud to the Edge. Computer Research and Development, vol. 57, no. 01, pp. 53 -73, 2020.

[5] Chang, K.R., Hu, A.Q., Liu, Z.Z. Intelligent Elderly Care Design Scheme Based on Internet of Things Technology. Computer Products and Circulation, no. 10, pp. 175, 2019.

[6] Jiang, H. Research and Implementation of Smart Pension Medical Health System Based on Data Fusion, Nanjing University of Posts and Telecommunications, 2015. 